\title{
Preliminary Model for Scaling of Fourier Spectra of Strong Ground Motion Recorded on Kamchatka
}

\author{
A. G. Petukhin, ${ }^{1}$ A. A. Gusev,,$^{1,2}$ E. M. Guseva, ${ }^{1}$ E. I. Gordeev ${ }^{1}$ and \\ V. N. CHEBRov ${ }^{1}$
}

\begin{abstract}
To determine the average relationship among the Fourier spectrum of horizontal acceleration $F S A(f)$, moment magnitude $M_{W}$ and hypocentral distance $R$ for Kamchatka earthquakes, we analyzed 44 analog strong-motion records recorded here in 1969-1993. The records of acceleration and velocity meters were obtained at 11 rock to medium-ground sites from 36 earthquakes with $M_{W}=4.5-7.8$, at distances $R=30-250 \mathrm{~km}$ and depths $0-80 \mathrm{~km}$. Amplitude spectra $F S A(f)$ were calculated from digitized, baseline corrected records of 81 horizontal components, and then divided by instrumental transfer function. After smoothing the values were picked at a set of fixed frequencies. With the scarce amount of data at hand it was impossible to determine reliably the entire $\operatorname{FSA}\left(M_{W}, R \mid f\right)$ average trend surface. Hence we first performed distance equalization with distance corrections calculated on a theoretical basis, and thus reduced the observed data to the reference distance of $R_{0}=100 \mathrm{~km}$. The model of distance attenuation applied included point source decay terms $(1 / R$ plus attenuation specified by $Q(f)=250 f^{0.8}$ ) and finite source correction (using the formula for a disc-shaped incoherent source, its size depending on $M_{W}$ ); its general applicability was later checked by analysis of residuals. After reduction we determined the $F S A\left(M_{W}, R_{0} \mid f\right)$ vs. $M_{W}$ trends. To do this we employed a multiple regression procedure with ground type and station dummy variables. The $M_{W}$ dependence was assumed to consist of two linear branches intersecting at $M_{W}=6.5$. The result of multiple regression represents the first systematic description of spectral properties of destructive ground motion for Kamchatka earthquakes. The empirical $F S A$ vs. $M_{W}$ trend flattens as frequency increases. This flattening persists even between 3 and $16 \mathrm{~Hz}$, suggesting the decrease of source-related $f_{\max }$ with increasing magnitude.
\end{abstract}

\section{Introduction}

Since 1962 a small network of analog strong-motion instruments operated in Kamchatka. A few of the most interesting of the recorded strong-motion records and spectra have been published (see GUSEV et al., 1997a for references). Recently, GuSEV et al. (1997a) analyzed, for the first time, the bulk of the data obtained during these observations and determined the first version of the $A_{\max }\left(M_{W}, R\right)$

\footnotetext{
${ }^{1}$ Kamchatka Experimental and Methodological Seismological Department, Geophysical Survey, Russian Academy of Science (KEMSD GS RAS), Petropavlovsk-Kamchatsky, 683006, Russia.

${ }^{2}$ Institute of Volcanic Geology and Geochemistry, Far East Branch of Russian Academy of Science, Petropavlovsk-Kamchatsky, 683006, Russia. E-mail: gusev@emsd.iks.ru; ivgg@svyaz. kamchatka.su
} 
relationship for Kamchatka. Below we continue the analysis of strong-motion parameters for Kamchatka and determine the average regional relationship among amplitude Fourier spectrum of acceleration FSA, moment magnitude $M_{W}$ and hypocentral distance $R$ for a shallow earthquake.

Fourier spectrum $F S A$ is used in engineering seismology, mainly for stochastic modelling of strong-motion parameters (BOORE, 1983; GUSEV, 1983; VANMARKE, 1986). When combined with an envelope function it can be immediately used for simulation of accelerograms (BOORE, 1983). Fourier spectrum can also be used for reconstruction of earthquake source spectrum (moment rate spectrum) in its high-frequency part. One of the first studies of $\operatorname{FSA}(M, R)$ interrelationships from strong motions has been carried out by TRIFUNAC (1976) for western U.S.A., applying multiple regression analysis of observed data. KAMIYAMA and MATSUKAWA (1990) have done analogous work based on Fourier spectra of 228 strong-motion records in Japan. In addition to magnitude and distance their regression model included station variables, and they calculated the spectral station corrections for 10 stations with most abundant data. For eastern North America the $\operatorname{FSA}(M, R)$ relationship and then average source spectra were determined by Atkinson and Mereu (1992) and AtKinson (1993). Gusev (1990) made a crude estimate of Fourier spectrum of strong motion for Petropavlovsk-Kamchatsky for an expected future earthquake by means of analysis of Fourier spectra of several earthquakes with $M=7-8$ in Kamchatka and Japan. This work requires continuation and refinement.

The initial data that we use here are the same set of digitized and processed records as in (GUSEV et al., 1997a). The records have been accumulated by the efforts of several seismological organizations, namely by the Institute of Physics of the Earth (IPE) of the Academy of Science of the USSR (AS USSR), under V. V. Shteinberg, by the Pacific Seismic Expedition of IPE under S. A. Fedotov, by the seismic station 'Petropavlovsk' of IPE under L. G. Sinelnikova, by the Institute of Volcanology of the Far Eastern Branch of the AS USSR under V. D. Theofilaktov and by Kamchatka Experimental and Methodological Seismological Department under E. I. Gordeev and V. P. Mityakin.

\section{Data and their Primary Processing}

Strong-motion station network on Kamchatka is depicted on Figure 1. The two main types of analog instruments used are SSRZ-M accelerometers with electromagnetic damping and ISO-IIM galvanometric velocity meters. Detailed information regarding parameters of instruments used can be found in GUSEV et al. (1998, Table 2). Ground conditions at the stations are given in Table 1.

In the following we analyze only good-quality records obtained at either free-field stations or by instruments installed on the ground floor or in the basement 
of one or two-storey buildings. Some records have been considered unreliable during data processing and were rejected after thorough analysis. The probable sources of assumed errors are: (1) erroneous association of the record to an event in the catalog and (2) large error of hypocentral coordinates leading to significant errors of the value of the hypocentral distance.

The data processing procedure applied to the records consisted of the following steps.

1. Digitization of optical records by a hand-operated optical-mechanical digitizer (F004 system, typically, about 80 counts per second) or, for the recent data, using a 300-dpi optical scanner and semi-automatic digitization code, interactively

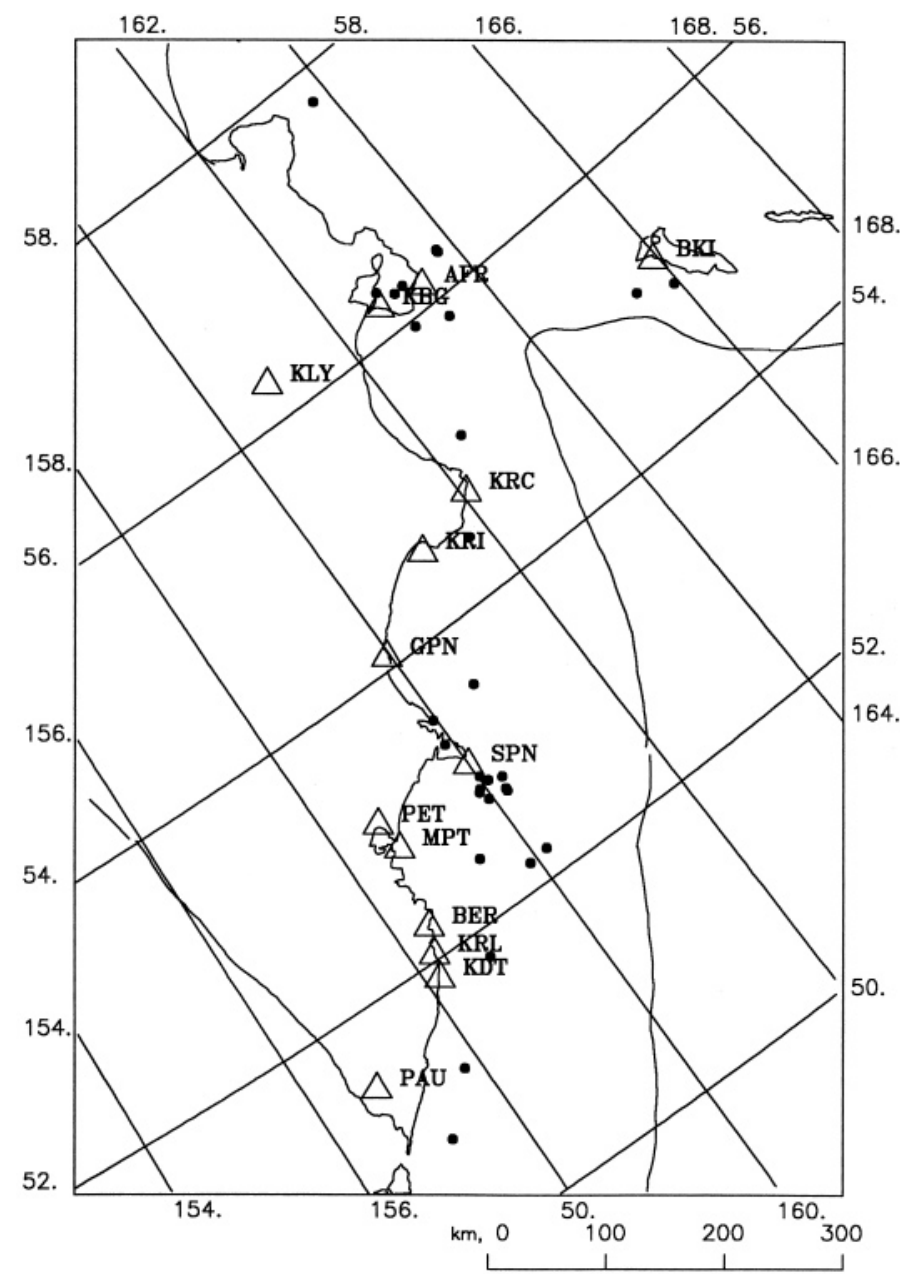

Figure 1

Strong-motion instrument network on Kamchatka. Triangles: instruments. Dots: epicenters of earthquakes given in Table 2. 
A. G. Petukhin et al. Pure appl. geophys.,

Table 1

Instrument location and ground conditions

\begin{tabular}{|c|c|c|c|c|c|}
\hline Station name & Code & Lat. & Long. & Ground type & $\begin{array}{l}\text { Ground } \\
\text { category }\end{array}$ \\
\hline Being & BKI & 55.2 & 166.0 & sandstone & rock \\
\hline Cape Africa & AFR & 56.2 & 163.4 & gravel + sand (paleolandslide & medium \\
\hline Krutoberegovo & KBG & 56.3 & 162.7 & gravel+ loam & medium \\
\hline Klyuchi & KLY & 56.3 & 160.9 & lava flow covered by sand + clay & medium \\
\hline Cape Kronotskiy & $\mathrm{KRC}$ & 54.7 & 162.1 & metamorphic schist & rock \\
\hline Kronoki & KRI & 54.6 & 161.2 & gravel + loam & medium \\
\hline Zhupanovo & GPN & 54.1 & 160.0 & lava flow & rock \\
\hline Shipunskiy & SPN & 53.1 & 160.0 & metamorphic schist & rock \\
\hline Lighthouse & MPT & 52.9 & 158.7 & & rock \\
\hline \multicolumn{6}{|l|}{ Petropavlovskiy } \\
\hline Paratunka & PRT & 53.0 & 158.2 & alluvium + gravel & medium \\
\hline Berezovaya & BER & 52.3 & 158.4 & $\begin{array}{l}\text { metamorphic schist covered by } \\
\text { gravel+ sand }\end{array}$ & rock \\
\hline Cape Kruglyi & KRL & 52.1 & 158.3 & tuff breccia & rock \\
\hline Khodutka & KDT & 51.8 & 158.1 & lava flow & rock \\
\hline \multicolumn{6}{|c|}{ Stations in Petropavlovsk-Kamchatsky } \\
\hline Petropavlovsk & PET & 53.0 & 158.6 & gabbro & rock \\
\hline Aerological st. & AER & 53.1 & 158.6 & pyroclastic deposits (paleolandslide) & medium \\
\hline Mt. Mishennaya & MSN & 53.0 & 158.6 & gabbro & rock \\
\hline Nikolskaya & NKS & 53.0 & 158.6 & green schist & rock \\
\hline
\end{tabular}

controlled at a computer display. In the most difficult cases, manual, pixel by pixel procedure of digitization was used.

2. Baseline correction. For most records we confined ourselves by subtraction of a straight line, fitted by the least-squares method, from the digitized data. For some records digitized with the scanner we subtracted the baseline of the accelerometer, recorded on the same film. This operation considerably reduces the low-frequency noise in spectra.

3. Cutting out segments with maximum amplitudes ( $S$-wave groups). Normally we have cut out the segment between $S$-arrival and the moment when the amplitude was equal to one half of the maximum value. This procedure has a minor effect on the level of the spectrum (as compared to processing the complete record) however it considerably improves the signal-to-noise ratio at the high-frequency part of a spectrum. A cosine taper with $10 \%$ duration was used at both ends of the cut segment.

4. Fourier spectrum calculation with FFT algorithm.

5. Instrument response correction in frequency domain, producing the ground acceleration spectrum. To calculate the instrument transfer function we used parameters of the instruments measured during regular calibration. Normally 
calibration was performed each $2-3$ years. The values of the damping constant for a few accelerometers with oil damping were set according to the manufacturer's specification.

6. Selecting the frequency band with an acceptable signal-to-noise-ratio. Typically spectra were reliable between 0.3 and $20 \mathrm{~Hz}$.

7. Smoothing and spectral level calculation. We employed the smoothing procedure with the window half-width of 0.1 in the logarithmic (LOG10) scale. After smoothing the values were picked at the frequencies of $0.5,1,2,3,5,10$ and $16 \mathrm{~Hz}$.

The software employed in data processing (steps 2-7) was designed by the authors; its description was published earlier (GUSEvA et al., 1989). The program for interactive digitization of scanned records was kindly provided by D. V. Droznin. Figure 2 shows an example of the original record and its Fourier spectrum.

The described procedure was applied to 81 horizontal strong-motion components, recorded from 36 earthquakes with $M_{W}=4.5-7.8$, at hypocentral distances
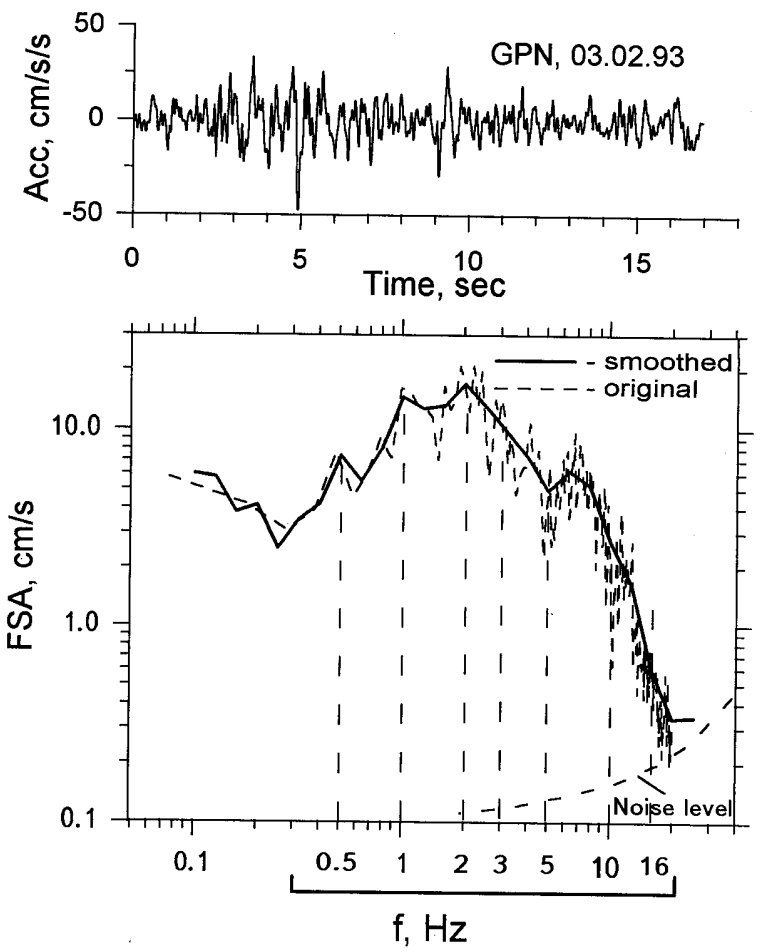

Figure 2

Example of Fourier spectrum calculation. The March 02, 1992 event recorded at station GPN, component EW, accelerometer SSRZ-M. The bar in the bottom shows the frequency band with reliable FSA estimates. 


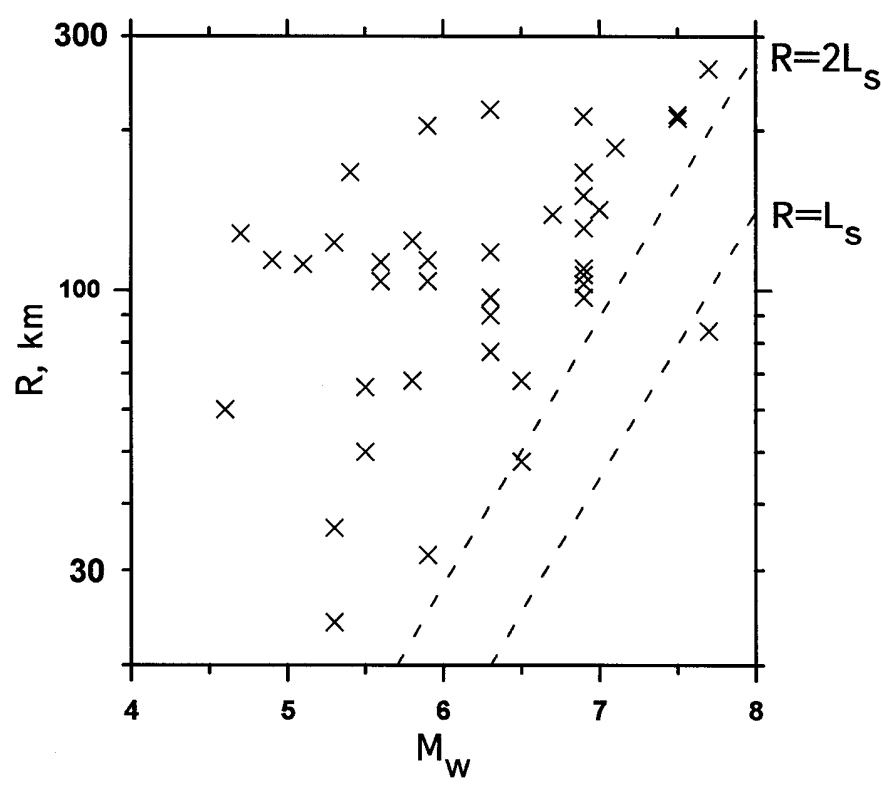

Figure 3

Distribution of processed records over magnitude and distance. Dashed lines denote the approximate boundary of the epicentral zone.

$R=30-250 \mathrm{~km}$ and depths $0-80 \mathrm{~km}$. On Figure 3 one can see the distribution of processed records over magnitude and distance. Epicentral locations are given in Figure 1. Distribution of epicenters does not reflect real seismicity; rather it shows the quality of maintenance of triggered instruments. Their work at remote, low populated regions was sometimes unreliable.

The earthquake origin times, coordinates and magnitudes are compiled in Table 2. For major earthquakes $M_{W}$ estimates are mainly based on the compilation by ZoBIN et al. (1988). For smaller ones the $M_{W}$ values used are (in the order of preference): based on Harvard CMT solution, estimated from $M_{L H}$ or other surface-wave magnitude, or, if no long- or medium-period data were available, estimated from the regional 'energy class' short-period magnitude $K^{F 68}$. To make conversions needed in the latter two cases, the empirical nonlinear intermagnitude relationships of GUSEV (1991) (specific for the region under study) were used. Coordinates and (when needed) magnitudes were taken from the regional earthquake catalog.

In Table 3 we give the values of acceleration spectra for the set of frequencies: $f_{K}=0.5,1,2,3,5,10$ and $16 \mathrm{~Hz}$ and also parameters of the records used in this study. Strong-motion instruments are colocated with regional seismic stations 
(BKI, KBG, KRI and PET), in such cases it was often possible to determine hypocentral distance $R$ from $t_{S}-t_{P}$, and this estimate was the preferred one. For other stations $R$ values were calculated from hypocentral coordinates. The gaps in Table 3 data are caused by low quality components or by unreliable results at particular frequencies. This table is the main result of the first part of the present study.

Table 2

List of earthquakes used

\begin{tabular}{|c|c|c|c|c|c|c|c|c|}
\hline \multirow[t]{2}{*}{ Date yy.mm.dd } & \multirow[t]{2}{*}{$T_{0}$ hh.mm.ss } & \multicolumn{2}{|c|}{ Epicenter } & \multirow[t]{2}{*}{$H, \mathrm{~km}$} & \multicolumn{4}{|c|}{ Magnitudes } \\
\hline & & Lat. & Long. & & $M_{\mathrm{LH}}$ & $M_{\mathrm{S}}$ & $K^{F 68}$ & $M_{W} *$ \\
\hline 69.11 .22 & 23.09 .35 & 57.76 & 163.75 & 30 & 7.7 & 7.3 & 14.4 & $7.7 t$ \\
\hline 71.12 .15 & 08.29 .55 & 55.85 & 163.35 & 25 & 7.8 & 7.8 & 16.0 & $7.7 z$ \\
\hline 71.12 .19 & 07.50 .23 & 55.95 & 162.90 & 0 & 5.8 & 5.3 & 11.9 & $5.9 l$ \\
\hline 73.03 .12 & 19.39 .21 & 50.80 & 157.20 & 70 & - & - & 14.4 & $6.7 k$ \\
\hline 77.11 .06 & 02.39 .38 & 53.50 & 159.96 & 60 & 4.6 & - & 13.2 & $5.3 h$ \\
\hline 77.12 .21 & 16.39 .36 & 52.19 & 159.90 & 39 & 5.3 & 5.0 & 12.8 & $5.6 h$ \\
\hline 79.06 .25 & 18.45 .52 & 52.74 & 160.20 & 31 & 4.0 & - & 13.1 & $4.7 l$ \\
\hline 80.02 .11 & 15.29 .47 & 53.30 & 159.90 & 57 & - & - & 12.7 & $5.1 k$ \\
\hline 80.11 .23 & 15.45 .03 & 52.44 & 159.42 & 20 & - & - & 11.8 & $4.6 k$ \\
\hline 80.12 .04 & 10.46 .27 & 52.21 & 160.17 & 26 & 5.6 & 5.2 & 12.8 & $5.4 h$ \\
\hline 81.02 .09 & 19.31 .30 & 54.94 & 165.94 & 20 & 5.1 & 4.9 & 12.2 & $5.3 h$ \\
\hline 81.06 .25 & 01.47 .56 & 52.85 & 159.90 & 42 & 4.2 & 4.3 & 12.5 & $4.9 l$ \\
\hline 82.03 .08 & 15.16 .31 & 52.89 & 160.08 & 38 & 4.2 & - & 12.1 & $5.1 h$ \\
\hline 82.04 .17 & 10.27 .12 & 54.44 & 161.72 & 42 & - & - & 10.9 & $4.6 k$ \\
\hline 82.05 .31 & 10.21 .21 & 55.07 & 165.48 & 56 & 6.5 & 6.4 & 14.7 & $6.5 h$ \\
\hline 83.04 .04 & 19.04 .23 & 52.95 & 160.02 & 40 & 5.7 & 5.5 & 13.3 & $5.9 h$ \\
\hline 83.08 .05 & 00.33 .47 & 52.87 & 159.93 & 41 & 4.7 & 4.7 & 12.6 & $5.5 h$ \\
\hline 84.12 .28 & 10.37 .47 & 56.28 & 163.77 & 5 & 7.5 & 7.0 & 14.5 & $6.7 h$ \\
\hline 85.03 .06 & 22.31 .52 & 55.09 & 162.48 & 46 & 6.0 & 5.4 & 14.3 & $5.9 h$ \\
\hline 85.05 .19 & 08.07 .48 & 53.54 & 160.65 & 40 & 5.6 & - & 13.7 & $5.8 h$ \\
\hline 85.08 .09 & 22.24 .03 & 56.25 & 163.10 & 0 & 4.9 & 4.5 & 12.3 & $5.3 l$ \\
\hline 85.08 .09 & 23.06 .60 & 56.24 & 162.95 & 7 & - & - & 11.5 & $4.9 k$ \\
\hline 87.02 .14 & 16.15 .14 & 56.33 & 162.76 & 16 & - & - & 10.9 & $4.5 k$ \\
\hline 87.10 .06 & 20.11 .36 & 52.85 & 160.25 & 34 & 6.6 & 6.3 & 13.9 & $6.5 h$ \\
\hline 89.05 .24 & 13.31 .18 & 56.30 & 163.77 & 39 & 6.4 & 6.1 & 13.5 & $6.3 h$ \\
\hline 92.03 .02 & 12.29 .38 & 52.76 & 160.20 & 20 & 7.1 & 6.8 & 14.6 & $6.9 h$ \\
\hline 92.03 .05 & 14.39 .11 & 52.77 & 159.95 & 31 & 6.2 & 6.1 & 14.0 & $6.3 h$ \\
\hline 93.06 .08 & 13.03 .37 & 51.20 & 157.80 & 40 & 7.4 & 7.3 & 14.9 & $7.5 h$ \\
\hline 93.11 .13 & 01.18 .07 & 51.79 & 158.83 & 40 & 7.1 & 7.0 & 14.6 & $7.0 h$ \\
\hline
\end{tabular}

* $M_{W}$ values are from different sources and each source is marked by the letter: $t$-tsunami magnitude of ABE (1979); $z$-based on $M_{0}$ estimates summarized in ZoBIN et al. (1988); $l$-estimated from $M_{L H}$ based on correlation (GUSEV, 1991); $k$-estimated from $K^{F 68}$ based on correlation (GUSEV, 1991); $h$-calculated from $M_{0}$ estimate of Harvard CMT. 
Table 3

Levels of the ground Fourier spectra of acceleration

\begin{tabular}{|c|c|c|c|c|c|c|c|c|c|c|c|c|c|c|}
\hline & \multirow[t]{2}{*}{ Date dd.mm.yy } & \multirow[t]{2}{*}{ Station code } & \multirow[t]{2}{*}{ Instr. type } & \multirow[t]{2}{*}{ Comp. } & \multirow[t]{2}{*}{$\Delta, \mathrm{km}$} & \multirow[t]{2}{*}{$R, \mathrm{~km}$} & \multirow[t]{2}{*}{$M_{W}$} & \multicolumn{7}{|c|}{$F S A(f), \mathrm{cm} / \mathrm{s}$} \\
\hline & & & & & & & & $f=0.5$ & 1 & 2 & 3 & 5 & 10 & 16 \\
\hline \multirow{2}{*}{1.} & 28.10 .60 & PET & SMR-3 & NS & 150 & 186 & 7.1 & 8.3 & 17.3 & 14.4 & 12.5 & - & - & - \\
\hline & & & & EW & & & & 11.4 & 12.0 & 17.3 & 27.5 & - & - & - \\
\hline \multirow{2}{*}{2.} & 22.11 .69 & BKI & ISO & NS & 260 & 261 & 7.7 & 3.63 & 1.73 & 1.44 & 1.0 & 1.0 & 1.38 & - \\
\hline & & & & EW & & & & 5.24 & 2.08 & 1.44 & 1.31 & 1.73 & 1.51 & - \\
\hline \multirow[t]{2}{*}{3.} & 15.12 .71 & KBG & UAR & NS & 80 & 84 & 7.7 & 31.6 & 26.3 & 19.9 & 12.3 & 8.7 & 2.88 & - \\
\hline & & & & EW & & & & 19.0 & 30.1 & 18.1 & 13.8 & 7.9 & 2.88 & - \\
\hline \multirow[t]{2}{*}{4.} & 19.12 .71 & KBG & UAR & NS & 32 & 32 & 5.9 & 6.0 & 3.63 & 2.29 & 2.18 & 2.18 & 0.57 & 0.25 \\
\hline & & & & EW & & & & - & 4.57 & 2.51 & 1.99 & 2.19 & 0.46 & - \\
\hline \multirow[t]{2}{*}{5.} & 12.03 .73 & PET & ISO & NS & 130 & 139 & 6.7 & 0.22 & 0.57 & 1.73 & 0.72 & 0.35 & 0.2 & - \\
\hline & & & & EW & & & & 0.22 & 0.63 & 1.65 & 0.55 & 0.41 & 0.19 & - \\
\hline \multirow[t]{2}{*}{6.} & 06.11 .77 & PET & ISO & NS & 107 & 123 & 5.3 & 0.087 & 0.18 & 0.48 & 0.42 & 0.33 & - & - \\
\hline & & & & EW & & & & 0.087 & 0.18 & 0.72 & 0.5 & 0.4 & - & - \\
\hline \multirow[t]{2}{*}{7.} & 21.12 .77 & PET & ISO & NS & 70 & 113 & 5.6 & 0.12 & 0.1 & 0.36 & 0.55 & 0.46 & 0.32 & 0.21 \\
\hline & & & & EW & & & & 0.17 & 0.38 & 0.36 & 0.91 & 0.87 & 0.38 & 0.22 \\
\hline \multirow[t]{2}{*}{8.} & 25.06 .79 & PET & ISO & NS & 80 & 128 & 4.7 & 0.03 & 0.1 & 0.35 & 0.4 & 0.4 & 0.16 & 0.1 \\
\hline & & & & EW & & & & 0.07 & 0.15 & 0.48 & 0.57 & 0.4 & 0.16 & 0.1 \\
\hline \multirow[t]{2}{*}{9.} & 11.02 .80 & PET & ISO & NS & 87 & 104 & 5.6 & 0.14 & 0.21 & 0.59 & 0.4 & 0.63 & 0.38 & 0.2 \\
\hline & & & & EW & & & & 0.12 & 0.4 & 0.55 & 0.79 & 0.6 & 0.27 & 0.15 \\
\hline \multirow[t]{2}{*}{10.} & 04.12 .80 & PET & ISO & NS & 140 & 167 & 5.4 & 0.55 & 0.5 & 0.83 & 0.72 & 0.23 & 0.14 & - \\
\hline & & & & EW & & & & 0.69 & 0.48 & 0.5 & 0.2 & 0.14 & 0.13 & - \\
\hline 11. & 09.02 .81 & BKI & SSRZ & NS & 30 & 36 & 5.3 & - & 2.29 & 3.16 & 3.63 & 3.63 & 5.75 & 1.0 \\
\hline \multirow[t]{2}{*}{12.} & 25.06 .81 & PET & ISO & NS & 85 & 114 & 4.9 & 0.06 & 0.07 & 0.26 & 0.32 & 0.26 & 0.13 & - \\
\hline & & & & EW & & & & 0.14 & 0.16 & 0.63 & 0.46 & 0.26 & 0.17 & - \\
\hline \multirow{3}{*}{13.} & 08.03 .82 & PET & ISO & NS & 110 & 112 & 5.1 & 0.04 & 0.23 & 0.5 & 0.66 & 0.51 & 0.15 & - \\
\hline & & & & EW & & & & 0.08 & 0.23 & 0.57 & 0.66 & 0.51 & 0.15 & - \\
\hline & 17.04 .82 & KRI & ISO & EW & 43 & 60 & 4.6 & 0.22 & 0.2 & 0.6 & 0.95 & 1.5 & 2.5 & 3.7 \\
\hline 15. & 31.05 .82 & BKI & SSRZ & NS & 38 & 68 & 6.5 & 10.0 & 5.24 & 7.24 & 4.57 & 7.24 & 6.91 & 2.88 \\
\hline \multirow[t]{2}{*}{16.} & 04.04 .83 & PET & ISO & NS & 90 & 104 & 5.9 & 0.48 & 0.66 & 1.1 & 1.0 & 0.93 & 0.52 & - \\
\hline & & & & EW & & & & 0.6 & 0.91 & 1.1 & 1.0 & 0.87 & 0.35 & - \\
\hline 17. & - & KRI & ISO & EW & 200 & 204 & - & 0.16 & 0.55 & 0.95 & 1.9 & 3.5 & 3.0 & 2.3 \\
\hline 18. & 05.08 .83 & SPN & ISO & EW & 29 & 50 & 5.5 & 0.3 & 0.46 & 1.1 & 1.2 & 1.0 & 0.69 & - \\
\hline 19. & 28.12 .84 & KBG & SSRZ & EW & 66 & 66 & 6.7 & 73.5 & 68.6 & 64.1 & 24.4 & 24.0 & 4.66 & 3.48 \\
\hline 20. & 06.03 .85 & KRI & ISO & NS & 104 & 114 & 5.9 & 0.83 & 1.2 & 1.0 & 1.73 & 2.5 & 3.8 & $\begin{array}{l}1.80 \\
1.8\end{array}$ \\
\hline & & & & EW & & & & 0.83 & 1.0 & 1.9 & 2.6 & 5.25 & 5.0 & 2.75 \\
\hline 21. & 19.05 .85 & SPN & ISO & EW & 55 & 68 & 5.8 & 1.0 & 2.0 & 3.16 & 5.24 & 2.18 & 1.15 & 1.0 \\
\hline 22. & - & KRI & ISO & NS & 117 & 124 & - & 0.23 & 1.05 & 0.63 & 1.07 & 4.8 & 3.16 & 4.8 \\
\hline & & & & EW & & & & 1.0 & 0.5 & 0.76 & 1.07 & 4.8 & 3.8 & 3.63 \\
\hline 23. & 09.08 .85 & KBG & SSRZ-M & NS & 24 & 24 & 5.3 & 2.47 & 10.3 & 9.18 & 9.54 & 14.2 & 29.8 & 4.38 \\
\hline & & & & EW & & & & 5.19 & 12.2 & 7.43 & 13.0 & 14.5 & 34.7 & 5.63 \\
\hline
\end{tabular}




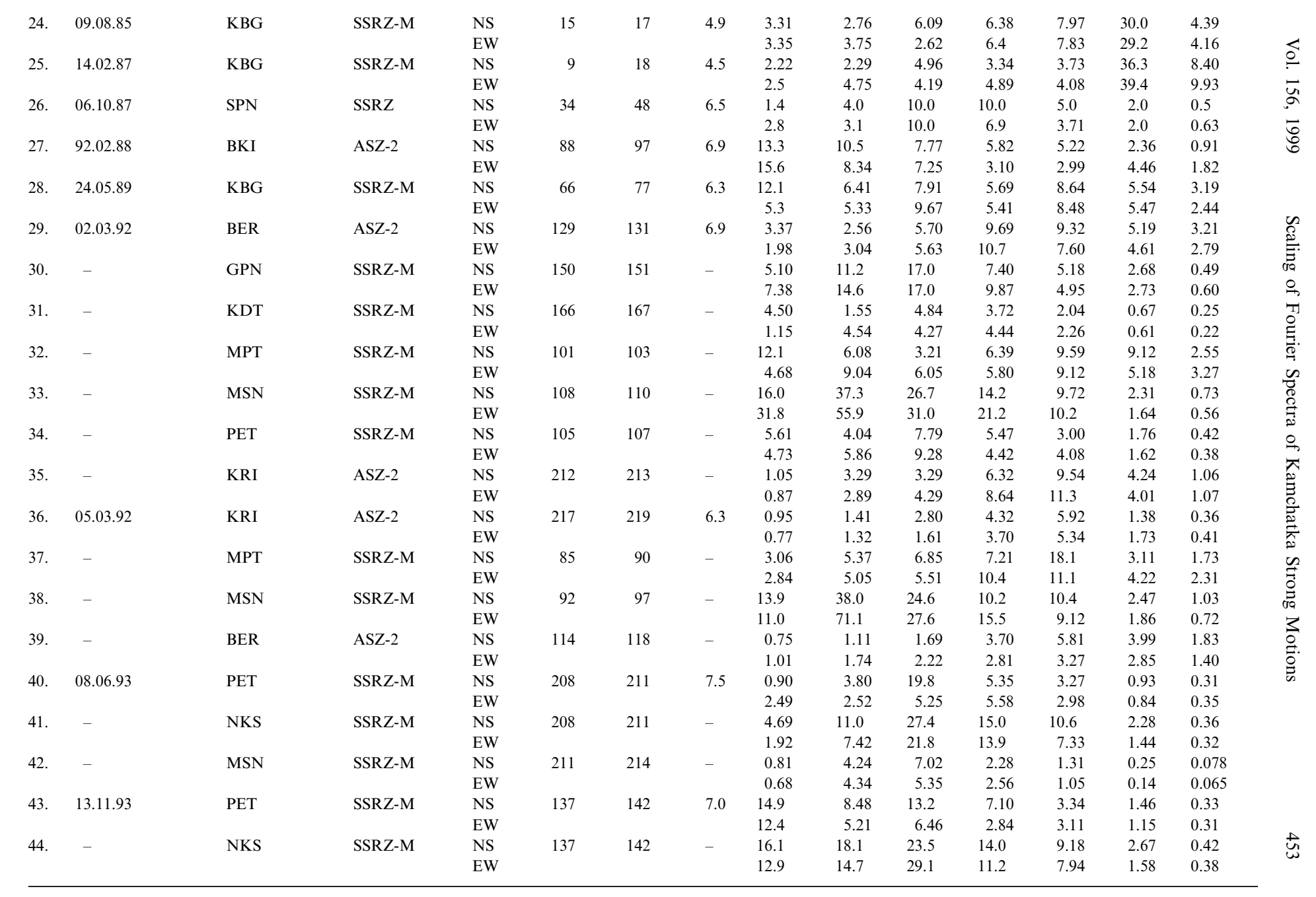




\section{Data Analysis: General Approach and Distance Reduction}

In regions with sufficiently abundant strong-motion records, such as western U.S.A. or Japan, average relationships between parameters of strong motion, magnitude, distance and other relevant parameters are usually determined on a purely empirical basis. In our case, however, data are too scarce for a reliable analysis of this kind. Instead, we will use a semi-empirical method: at first we reduce the data to the standard distance $R_{0}$, employing a theoretical relationship, and then relate these reduced Fourier spectrum levels at particular frequencies $f_{K}$ to magnitude by means of multiple regression procedure. It should be noted that there are no reliable, theoretical models for spectrum versus magnitude dependence at high frequencies, whereas the distance dependence is known from small earthquake data with some confidence. Therefore we represent an observed value of Fourier spectrum of acceleration FSA at a particular frequency by means of the following model:

$$
\log F S A_{i}=\log F S A^{E}\left(M_{W_{i}}, R_{0}\right)-\operatorname{Att}\left(M_{W_{i}}, R_{0}\right)+\operatorname{Att}\left(M_{W_{i}}, R_{i}\right)+\varepsilon_{i}
$$

where $F S A^{E}$ is the target empirical spectral model for the specific distance $R_{0}$, Att is the theoretical distance decay function, defined in detail in the next section, and $\varepsilon_{i}$ is a deviation of an individual value from the average trend.

The theoretical Att is defined as the sum of three terms that specify, correspondingly, the effects of the following three factors: geometrical spreading, attenuation and source finiteness:

$$
\operatorname{Att}\left(M_{W}, R\right)=\log (1 / R)+\log e^{-\pi f R / Q(f) c_{s}}+\log \left(\left(\frac{R^{2}}{R_{s}^{2}} \ln \left(\frac{R^{2}+R_{s}^{2}}{R^{2}+R_{\mathrm{coh}}^{2}}\right)\right)^{1 / 2}\right) .
$$

The first two terms define point source amplitude factor. Attenuation effect is specified by the $Q$-factor: $Q(f)=250 f^{0.8}$ if $f>1 \mathrm{~Hz}$, and $Q(f)=250$ if $f \leq 1 \mathrm{~Hz}$ (estimated previously from local small earthquakes data (ABYBAKIROV and GUSEV, 1990)). The third term is the source finiteness factor. It is calculated for a disc-shaped, noncoherent radiator (GUSEV, 1983), with magnitude-dependent effective source radius $R_{S}$. $R_{\text {coh }}$ is the coherence radius of an assumedly incoherent (random) high-frequency earthquake source. It also can be viewed as the size of a smallest subsource. Following GuSEv (1983) we accept here $R_{\text {coh }}=1 \mathrm{~km}$.

Based on preliminary estimates which used both strong motion and small earthquake data, we considered the $1 / R$ geometric spreading as an acceptable first approximation between 30 and $250 \mathrm{~km}$ for $S$-waves in the Kamchatka region (no $L g$ group is observed here at $R>100 \mathrm{~km}$ ). Later we will justify this assumption by means of analysis of residuals of our model vs. distance. The source finiteness factor describes the near-source saturation: the level of Fourier spectrum near an extended source of a large earthquake will be below the level expected for an equivalent point source. Use of the formula after GuSEv (1983) to allow for this effect seems 
justified for the present study. Its applicability for a strong-motion FSA data analysis has been verified by TRIFUNAC and LEE (1990) in a special study.

The theoretical $\operatorname{Att}\left(M_{W}, R\right)$ model described above is further used in two ways: first, for data analysis, to reduce data to the distance $R_{0}$; and second, in constructing the average resulting relationship, where we need to extrapolate estimated average reduced spectra from $R_{0}$ to other distances, thus obtaining the entire $F S A\left(M_{W}, R \mid f\right)$ trend surface. In the first mode the source finiteness correction factor has in fact minor effect, as there are only two records in the near zone (see Fig. 3). In the second mode this factor is rather significant as it provides realistic extrapolation of spectral levels defined at $R_{0}=100 \mathrm{~km}$ into near-source zones of large earthquakes.

Of course, for subduction-zone earthquakes, when most source zones are situated under the sea bottom, it is extremely difficult to directly verify such an extrapolation. We nevertheless consider our approach reasonable because, at odds with many empirical models of near-source saturation, it is theoretically founded; it also successively predicts near-source saturation for an earthquake on land (TRIFUNAC and LEE, 1990).

The value of effective source radius $R_{S}$ was calculated from its length $L_{S}$ according to the simple estimate (GUSEV, 1983): $R_{s} \approx 0.4 L_{s}$, valid for the value of source area aspect ratio of $2-3$, acceptable for Kamchatka earthquakes. To calculate $L_{s}$ we used semi-empirical dependence $L_{s}$ vs. $M_{W}$, assuming an exact geometric similarity of sources: $L_{s} \sim\left(S_{s}\right)^{0.5}$ and $S_{s} \sim M_{W}$, where $S_{s}$ is the area of rectangular rupture; hence the source length can be calculated with the formula:

$$
\log L_{s}=0.5 M_{W}-C_{L} \text {. }
$$

The constant $C_{L}=1.85$ was determined in compilation (Gusev and Melnikova, 1990) as a good approximation for subduction zone earthquakes of the Pacific. This value is quite consistent with a few Kamchatkan data and was accepted below.

The described reduction technique is in fact the particular version of the multiple-use procedure of GUSEV and PETUKHIN (1996). Recently the latter was applied to peak acceleration data (GUSEV et al., 1997a) and yielded attenuation relations that compare quite well with empirical trends of FUKUSHIMA and TANAKA (1990) based on a considerably larger volume of data.

\section{Multiple Regression Analysis of Reduced Data}

As explained above, the first step in determination of the average $F S A\left(M_{W}, R \mid f\right)$ relationship was the reduction of individual $F S A\left(M_{W_{i}}, R_{i} \mid f_{K}\right)$ data to the standard distance $R_{0}$. The particular value of $R_{0}=100 \mathrm{~km}$ was accepted, as it is near the middle of the range of distances in Table 3 (in logarithmic scale). The reduced values, denoted $F S A_{0}\left(M_{W} \mid f_{K}\right) \equiv F S A\left(M_{W}, R_{0} \mid f_{K}\right)$, can be expressed by the formula deduced from (1): 
$\log F S A_{0}\left(M_{W_{i}} \mid f_{K}\right)$
$\quad=\log F S A\left(M_{W_{i}}, R_{i} \mid f_{K}\right)-\operatorname{Att}\left(M_{W_{i}}, R_{i} \mid f_{K}\right)+\operatorname{Att}\left(M_{W_{i}}, R_{0} \mid f_{K}\right)$.

At the second stage of data interpretation we determined empirical trends $F S A^{E}\left(M_{W} \mid f_{K}\right)$ of the reduced data $F S A_{0}$ by means of multiple regression analysis. The parameters included within this regression were: $M_{W}$ and ground type or individual station. Such an approach does not appear systematic; it seems more logical to seek station corrections for each station (relative to some reference site). However, in our data set most earthquakes are recorded by only a single instrument, and even worse, many stations maintain only a few records. For these stations, estimates of station correction terms will inevitably be highly unreliable, biased by peculiarities of spectra of individual earthquakes. To avoid this, we seek site corrections only for the three stations that have abundant records; incidentally, they are also characterized by clear site anomalies. This decision is based on our previous analysis of peak accelerations (GUSEV et al., 1997a) and on a preliminary analysis of Fourier spectra (GUSEv et al., 1997b). These two studies have revealed prominent individual station anomalies for the three stations, whereas two of these stations supplied most of the volume of medium-ground data. For these reasons we divided the data set into the following four groups: (1) stations with rock ground (taken as the reference, with station correction fixed as zero); (2) station Krutoberegovo (KBG, medium ground); (3) station Petropavlovsk (PET, very hard rock) and (4) station Kronoki (KRI, medium ground).

In a limited number of cases (earthquakes of 19.05.1985, 02.03.1992, 05.03.1992, 08.06.1993 and 13.11.1993) we have more than one record from each earthquake (see Table 3). To eliminate possible bias related to increased weights of individual earthquakes when recorded at several stations, data for each of these events were averaged beforehand. Note that very limited information on the distance attenuation that can be extracted from these scarce single-event, multiple-distance data do not contradict the assumed attenuation law.

Subsequently we determined empirical trends of the logarithm of Fourier spectrum versus magnitude for rock ground: $\log F S A_{\text {rock }}^{E}\left(M_{W} \mid f=f_{K}\right)$, and also the spectral corrections for three other groups with respect to the rock ground.

The $F S A^{E}$ vs. $M_{W}$ relationship was expected to be nonlinear. Initially we considered the possibility of representing the magnitude trend by quadratic parabola. However, the parabolic representation sometimes behaves unstable near the boundaries of the data range, especially in our case of a limited amount of data (e.g., it may show a physically meaningless maximum around $M_{W}=8$ ). Hence it was judged to be inferior as compared to another (also three-parameter) model, consisting of two linked linear segments. The corner point linking the two segments was chosen at $M_{W}=6.5$. This value is near the middle of the range of observed event magnitudes. The final empirical model applied for fitting of the reduced data is: 
Table 4

Results of the multiple regression analysis by formula (5)

\begin{tabular}{lllrrrrr}
\hline$f_{K}, \mathrm{~Hz}$ & \multicolumn{1}{l}{$\beta_{1}$} & \multicolumn{2}{c}{$\beta_{2}$} & \multicolumn{1}{c}{$c_{1}$} & \multicolumn{1}{c}{$c_{2}$} & \multicolumn{1}{c}{$c_{3}$} & $\sigma_{\varepsilon K}$ \\
\hline 0.5 & $0.93 \pm 0.12$ & $0.44 \pm 0.15$ & $0.53 \pm 0.32$ & $0.97 \pm 0.16$ & $-0.17 \pm 0.14$ & $0.44 \pm 0.16$ & 0.38 \\
1 & $0.86 \pm 0.09$ & $0.54 \pm 0.12$ & $0.66 \pm 0.27$ & $0.78 \pm 0.12$ & $-0.16 \pm 0.10$ & $0.62 \pm 0.12$ & 0.30 \\
2 & $0.78 \pm 0.10$ & $0.60 \pm 0.13$ & $0.77 \pm 0.30$ & $0.46 \pm 0.13$ & $0.02 \pm 0.11$ & $0.51 \pm 0.13$ & 0.32 \\
3 & $0.61 \pm 0.09$ & $0.55 \pm 0.12$ & $0.68 \pm 0.29$ & $0.26 \pm 0.12$ & $-0.13 \pm 0.10$ & $0.65 \pm 0.12$ & 0.30 \\
5 & $0.57 \pm 0.08$ & $0.54 \pm 0.11$ & $0.58 \pm 0.23$ & $0.10 \pm 0.11$ & $-0.18 \pm 0.09$ & $0.86 \pm 0.11$ & 0.27 \\
10 & $0.40 \pm 0.08$ & $0.53 \pm 0.11$ & $0.28 \pm 0.12$ & $-0.41 \pm 0.11$ & $-0.34 \pm 0.10$ & $0.70 \pm 0.11$ & 0.27 \\
16 & $0.15 \pm 0.01$ & $0.39 \pm 0.16$ & $-0.19 \pm 0.21$ & $-0.45 \pm 0.14$ & $-0.27 \pm 0.13$ & $0.66 \pm 0.13$ & 0.30 \\
\hline
\end{tabular}

$$
\begin{aligned}
& \log F S A_{0}\left(M_{W} \mid f_{K}\right)=c_{0}\left(f_{K}\right)+\sum_{i=1}^{3} c_{i}\left(f_{K}\right) \cdot \delta_{i}+\varepsilon_{K}\left(f_{K}\right) \\
& + \begin{cases}\beta_{1}\left(f_{K}\right)\left(M_{W}-6.5\right) & \text { at } M_{W}<6.5 \\
\beta_{2}\left(f_{K}\right)\left(M_{W}-6.5\right) & \text { at } M_{W}>6.5\end{cases}
\end{aligned}
$$

where $M_{W}$ is moment magnitude, $c_{0}$ is the constant term, $f_{K}$ is the $K$-th frequency, $\varepsilon_{K}$ is the random error, assumed to have zero mean and variance $\sigma_{\varepsilon K}^{2}$. The $\delta_{i}$ symbol denotes a "dummy" variable: $\delta_{1}=1$ if an individual datum belongs to KBG station, otherwise $\delta_{1}=0 ; \delta_{2}$ in a similar manner is associated with PET station; and $\delta_{3}$ is associated with KRI. If the datum belongs to another rock ground station then all $\delta_{i}=0$ (no non-rock stations were used except for KRI and KBG). Therefore $c_{i}, i=1,2,3$ are the correction factors for KBG, PET and KRI with respect to the rock ground stations. The parameters $c_{0}, c_{i}, \beta_{1}, \beta_{2}$ are unknowns, to be sought for in the regression.

It should be mentioned that the use of the described model yields a dramatic reduction of residual error as compared to our preliminary study (GUSEv et al., 1997b), in which we used the simple linear law and no dummy variables. For a representative case of $2 \mathrm{~Hz}$ frequency, $\sigma_{\varepsilon}$ was reduced from 0.45 to 0.30 .

\section{The Average Relationship Between Fourier Spectrum, Magnitude and Distance for Kamchatka}

The results of the first processing step, i.e., of the data reduction to the standard distance of $100 \mathrm{~km}$, are shown for each frequency in Figure 4. On these plots we also show the results of fitting of the model (5). The parameters of this model for each $f_{K}$ are presented in Table 4 .

The residuals of data fitting by the described model were further used to check the applicability of the assumed distance attenuation model (Fig. 5). Parameters of 


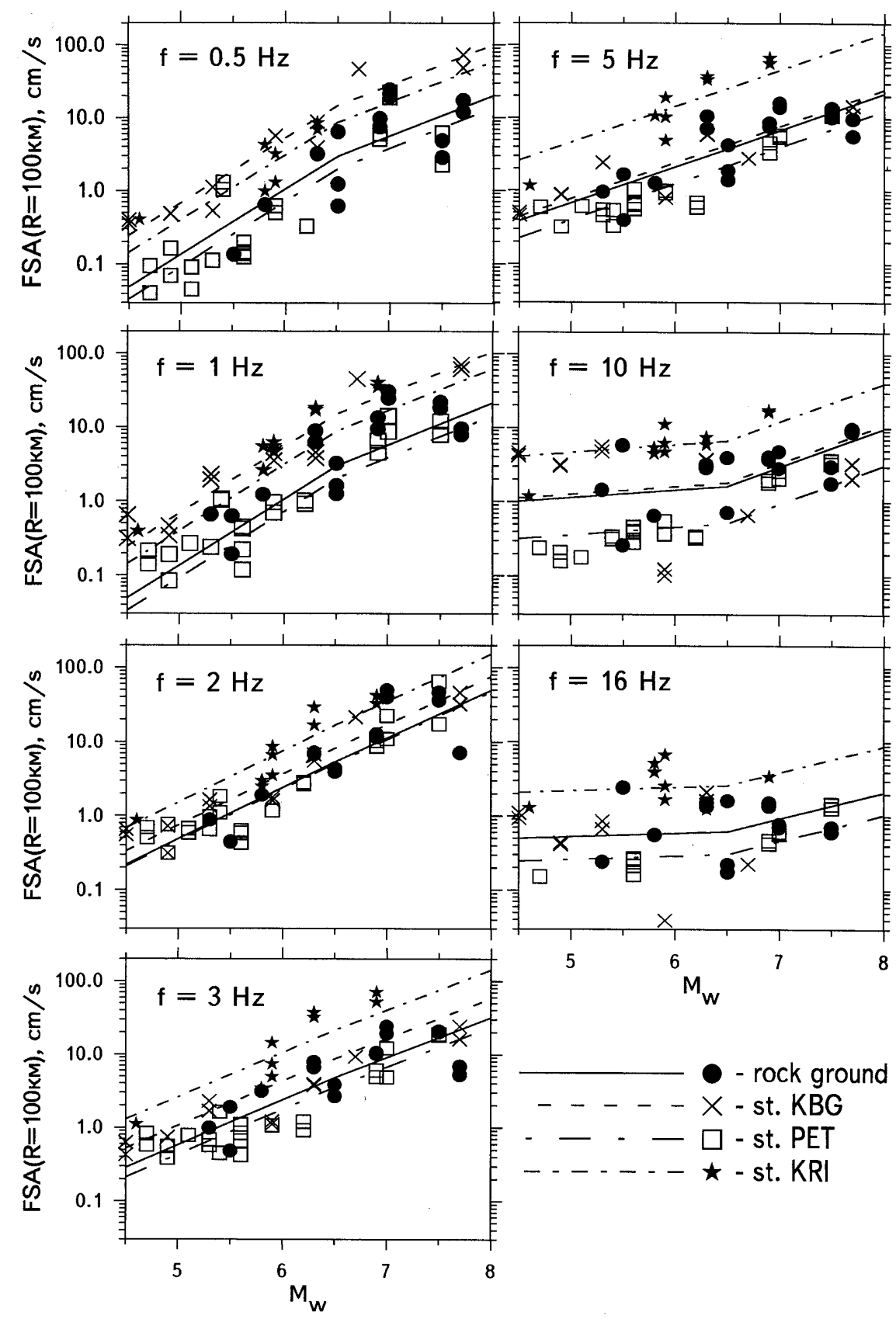

Figure 4

$F S A\left(f_{K}\right)$ values reduced to the constant $R_{0}=100 \mathrm{~km}$. Segmented lines are estimated trends of the model (5). For the coefficients see Table 4. Symbols denote the four data groups. 
linear and quadratic regressions of the residuals vs. distance are given in Table 5 . From Figure 5 one can discern no general trends in residuals. Also, all the estimates of the slope for the residual vs. distance trend (given in Table 5) are insignificant. Nevertheless, the systematically negative (though individually insignificant) values of this slope suggest that some slight deviations from the accepted model are present. From these slope values we could estimate the refined value of $Q_{0}$, equal to 295. Even more important is the insignificance of quadratic terms in Table 5; it shows the applicability of $1 / R^{n}$ geometric spreading model (with $n \approx 1$ ) for the whole range $R=30-250 \mathrm{~km}$ on Kamchatka. The only formal exclusion is the case of $f_{K}=10 \mathrm{~Hz}$. We treat this fact as a random fluctuation, that is, as a meaningless peculiarity of our data set.

Based on the estimated model, we calculated expected average Fourier spectra for rock ground and stations KBG, PET and KRI at $R_{0}=100 \mathrm{~km}$ and $M_{W}=8$, shown in Figure 6. Average expected Fourier spectra for rock at $R_{0}=100 \mathrm{~km}$ and $M_{W}=5,6,7$ and 8 are shown in Figure 7.

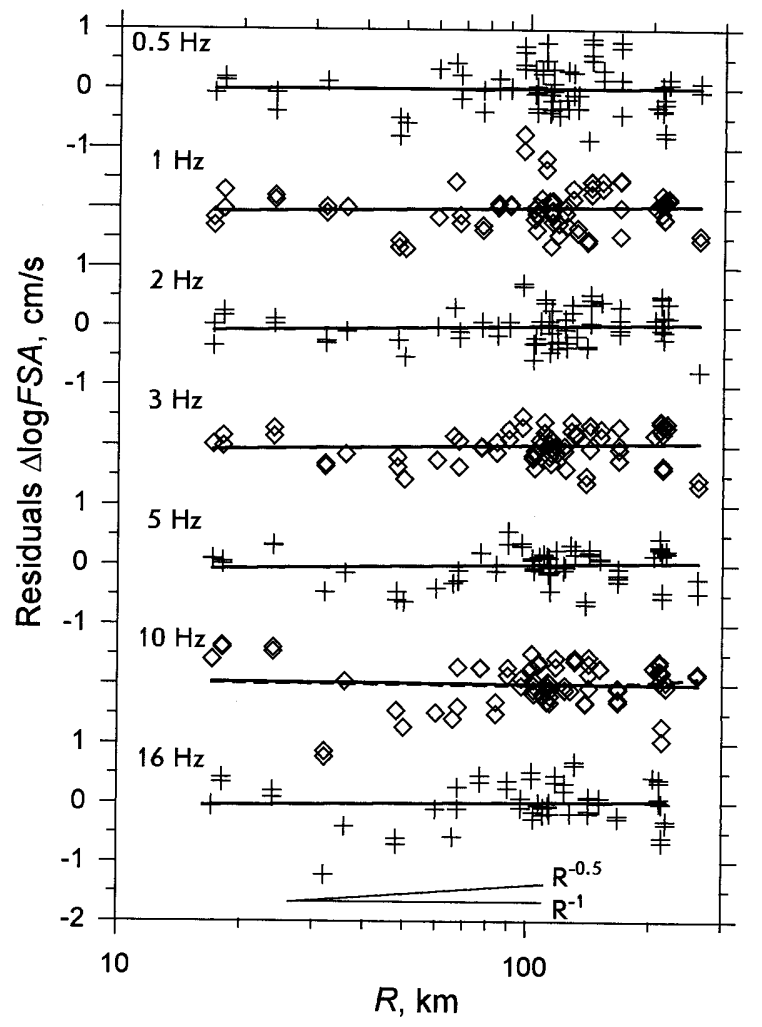

Figure 5

Residuals of data vs. distance for set of frequencies. Straight lines show least-squares fits. Sides of the sharp angle show expected slopes of average residual in case of real attenuation are $1 / R$ or $1 / R^{0.5}$. 
Table 5

Parameters of regressions of residuals $\Delta$ vs. $R$

\begin{tabular}{|c|c|c|c|c|c|c|c|c|}
\hline \multirow[t]{2}{*}{$f, \mathrm{~Hz}$} & \multicolumn{2}{|c|}{$\begin{array}{l}\text { Linear regression } \\
\Delta \log F S A=a \\
+b \cdot R\end{array}$} & \multicolumn{2}{|c|}{$\begin{array}{l}\text { Linear regression } \\
\Delta \log F S A=a \\
+b \cdot \log R\end{array}$} & \multicolumn{4}{|c|}{$\begin{array}{l}\text { Quadratic regression } \Delta \log F S A=a+ \\
b \log R+c \cdot(\log R)^{2}\end{array}$} \\
\hline & $b \cdot 10^{3}$ & $\sigma_{b} \cdot 10^{3}$ & $b$ & $\sigma_{b}$ & $b$ & $\sigma_{b}$ & $c$ & $\sigma_{c}$ \\
\hline 0.5 & 0.03 & 0.75 & 0.019 & 0.17 & 1.6 & 3.3 & -0.43 & 0.44 \\
\hline 1 & 0.21 & 0.7 & 0.088 & 0.15 & 0.45 & 2.4 & -0.1 & 0.4 \\
\hline 2 & 0.4 & 0.6 & 0.1 & 0.13 & -0.30 & 1.0 & 0.11 & 0.35 \\
\hline 3 & 0.48 & 0.54 & 0.1 & 0.11 & -0.72 & 1.2 & 0.22 & 0.3 \\
\hline 5 & 0.43 & 0.55 & 0.099 & 0.11 & -0.6 & 1.1 & 0.19 & 0.3 \\
\hline 10 & 0.33 & 0.76 & -0.032 & 0.16 & -3.8 & 3.0 & 1.0 & 0.4 \\
\hline 16 & 0.1 & 0.8 & 0.06 & 0.16 & 0.05 & 10.5 & 0.002 & 0.46 \\
\hline
\end{tabular}

Thus we have determined the empirical spectral model $F S A_{\text {rock }}^{E}\left(M_{W} \mid f\right)$ for rock ground conditions. To determine the average regional relationship between Fourier spectra at particular frequencies, moment magnitude and distance, we should pass to another arbitrary distance $R$. To make this we use the theoretical model $\operatorname{Att}\left(M_{W}, R\right)$ again:

$$
\log F S A\left(M_{W}, R \mid f\right)=\log F S A^{E}\left(M_{W} \mid f\right)-\operatorname{Att}\left(M_{W}, R_{0} \mid f\right)+\operatorname{Att}\left(M_{W}, R \mid f\right) .
$$

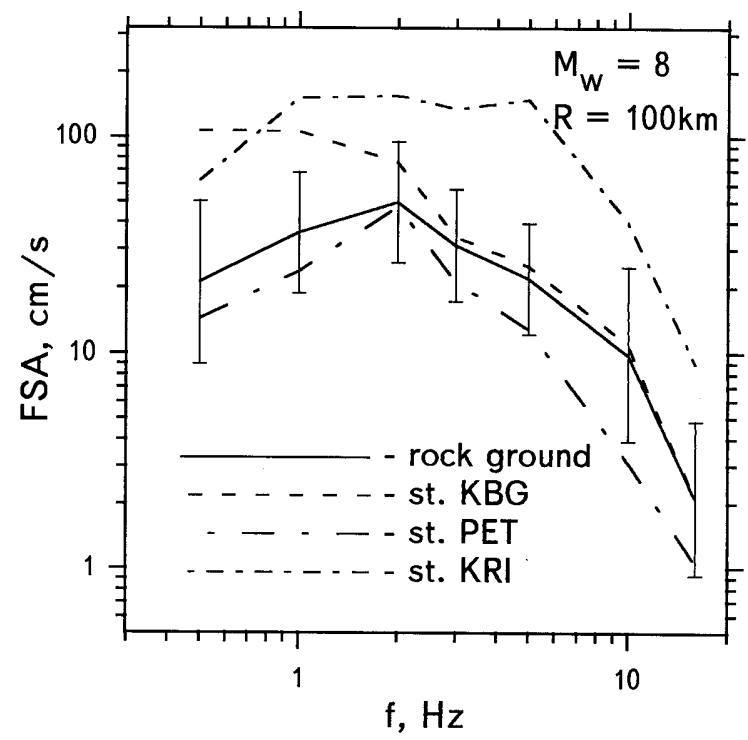

Figure 6

Estimated average Fourier spectra at $M_{W}=8$ and $R=100 \mathrm{~km}$ for four data groups. Error bars denote standard deviations of data. 


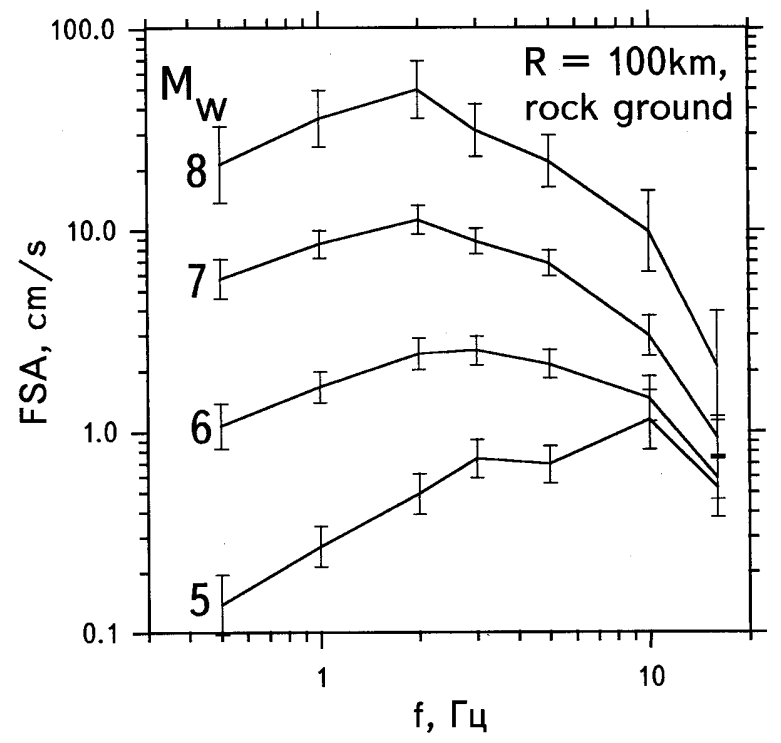

Figure 7

Estimated average Fourier spectra at $R=100 \mathrm{~km}$, on rock, for $M_{W}=5,6,7$ and 8 . Error bars-rmsprediction errors of the fitted regression model.

The result (for rock) is shown in Table 6 for a set of magnitudes, distances and frequencies.

In Figure 8 one can compare our results to those of other studies: average spectrum for Japan (KAMiYAma and MATSUKAwA, 1990), the spectrum recommended earlier for Petropavlovsk-Kamchatsky (GuSEv, 1990), and a recent spectrum estimate for California, based on most abundant data from ATKINSON and Silva (1997).

From the analysis of Table 4 and figures one can perceive the following:

1. With the use of multiple regression procedure with data divided into four groups one obtains the RMS scatter of FSA residuals of about 0.30 in $\log$ (base 10) units, which is typical for earlier similar studies, therefore the accepted regression scheme seems justified.

2. The coefficients $\beta_{1}(f)$ and $\beta_{2}(f)$ decrease with frequency. This is seen both in the $0.5-2 \mathrm{~Hz}$ range where this is expected from the BRUNE (1970) spectral model and also at higher frequencies where it is consistent with the GUSEV (1989) spectral model and inconsistent with the BRUNE (1970) model. The value of $\beta(f)$ in the range $2-5 \mathrm{~Hz}$ is slightly above the value $\beta=0.5$ expected from the Brune model (the difference is not significant). The decrease of $\beta(f)$ above $3 \mathrm{~Hz}$ may indicate the existence of a magnitude dependent, source-related $f_{\max }$ as discussed in GUSEV 
(1983). The inequality $\beta_{2}>\beta_{1}$ that is seen for the frequency range $5-16 \mathrm{~Hz}$ is unexpected. Its significance is low and we cannot consider it at present as the established one. It can be meaningful however because similar features are present in the empirical spectral model of TRIFUNAC (1989) and even more clear in the regression model of KAMIYAMA and MATSUKAWA (1990).

3. The absolute level of the estimated average spectrum for the rock ground is comparable with the level estimated earlier for Petropavlovsk-Kamchatsky for the same $R=80 \mathrm{~km}, M_{W}=7.15$ case (GUSEv, 1990) over most of the frequency range under discussion. The spectra for Japan and for Kamchatka are comparable in the range $0.5-2 \mathrm{~Hz}$. In the range $5-16 \mathrm{~Hz}$ the spectral level for Japan is approximately twice that for Kamchatka. Above $1 \mathrm{~Hz}$ both these spectra are greater than the average California spectrum.

4. The correction factor $c_{1}(f)$ for KBG station at low frequencies (see Fig. 5) is unusually large. The average amplitude of KBG spectrum at $f=0.5-1 \mathrm{~Hz}$ is five times above that for rock ground whereas they are practically equal at $f>3 \mathrm{~Hz}$. This result cannot be related to instability of the multiple regression procedure; it is evident in the initial data (see Fig. 4). No significant anomaly has been found for KBG in terms of peak acceleration amplitude: in GUSEv et al. (1997a) we found that $A_{\max }$ data for KBG do not differ from other medium-ground (or rock ground)

Table 6

Values of average Fourier spectra for Kamchatka $(\mathrm{cm} / \mathrm{s})$ for a set of magnitudes and distances (rock ground)

\begin{tabular}{|c|c|c|c|c|c|c|c|c|}
\hline \multirow[t]{2}{*}{$R, \mathrm{~km}$} & \multirow[t]{2}{*}{$M_{W}$} & \multicolumn{7}{|l|}{$f, \mathrm{~Hz}$} \\
\hline & & 0.5 & 1 & 2 & 3 & 5 & 10 & 16 \\
\hline \multirow[t]{4}{*}{25} & 5 & 0.68 & 1.39 & 2.65 & 4.11 & 3.95 & 6.97 & 3.88 \\
\hline & 6 & 4.80 & 8.55 & 12.94 & 13.84 & 12.25 & 8.74 & 3.71 \\
\hline & 7 & 23.52 & 40.09 & 55.07 & 44.07 & 35.59 & 16.38 & 5.40 \\
\hline & 8 & 61.19 & 118.50 & 171.27 & 110.77 & 80.45 & 38.11 & 8.61 \\
\hline \multirow[t]{4}{*}{50} & 5 & 0.30 & 0.64 & 1.20 & 1.83 & 1.75 & 3.02 & 1.45 \\
\hline & 6 & 2.32 & 3.95 & 5.88 & 6.25 & 5.47 & 3.83 & 1.60 \\
\hline & 7 & 12.16 & 19.74 & 26.85 & 21.32 & 16.98 & 7.67 & 2.49 \\
\hline & 8 & 39.44 & 73.01 & 104.4 & 66.96 & 48.14 & 22.35 & 4.97 \\
\hline \multirow[t]{4}{*}{100} & 5 & 0.14 & 0.27 & 0.49 & 0.73 & 0.68 & 1.14 & 0.53 \\
\hline & 6 & 1.06 & 1.65 & 2.40 & 2.50 & 2.14 & 1.45 & 0.59 \\
\hline & 7 & 5.67 & 8.43 & 11.18 & 8.72 & 6.77 & 2.96 & 0.93 \\
\hline & 8 & 20.94 & 35.35 & 49.07 & 31.02 & 21.75 & 9.76 & 2.10 \\
\hline \multirow[t]{4}{*}{200} & 5 & 0.06 & 0.09 & 0.16 & 0.23 & 0.21 & 0.32 & 0.14 \\
\hline & 6 & 0.44 & 0.58 & 0.79 & 0.80 & 0.65 & 0.41 & 0.16 \\
\hline & 7 & 2.39 & 2.97 & 3.72 & 2.80 & 2.08 & 0.84 & 0.25 \\
\hline & 8 & 9.21 & 13.01 & 17.13 & 10.44 & 6.97 & 2.91 & 0.59 \\
\hline
\end{tabular}




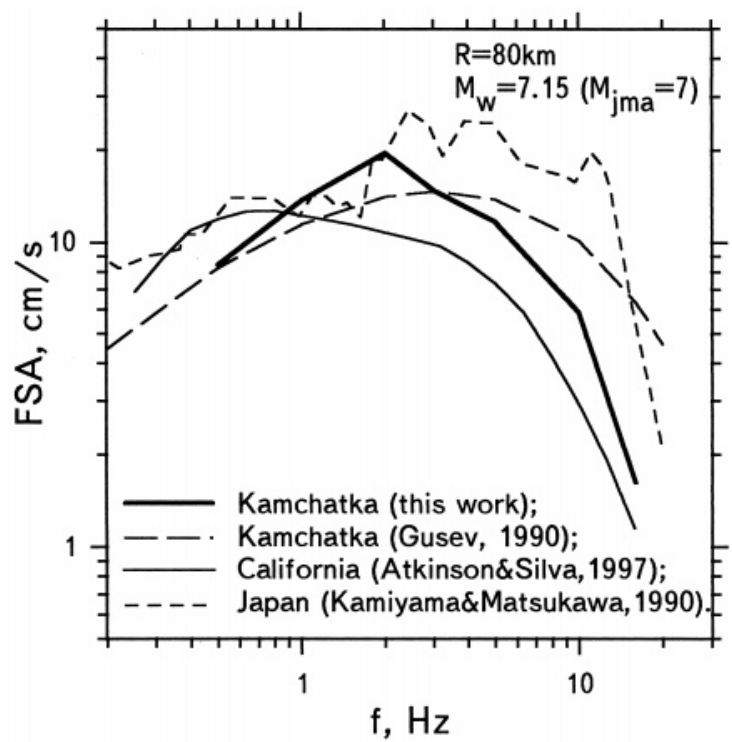

Figure 8

Average Fourier spectrum at $R=80 \mathrm{~km}$ and $M_{W}=7.15\left(M_{j m a}=7\right)$, rock ground, for Kamchatka (this work) compared to similar spectra calculation from regression formulas for Japan (KAMIYAMA and Matsukawa, 1990) and California (AtKinson and Silva, 1997). Also given is the expected Kamchatka spectrum from GuSEV (1990), adjusted to $M_{W}=7.15$.

stations. This fact agrees with the absence of any anomaly at higher $(f>3)$ frequencies. However, the same "normal" behavior is also seen for KBG in macroseismic data of several earthquakes which occurred around it. The revealed inconsistency needs further study; it may be resolved only if additional data from other stations and events will be used. At present one can only surmise. One possible cause may be that the estimation of spectral correction for KBG station is strongly biased by spectral differences between «northern» events, located near this station, and «southern» events. Another possible cause is the enhanced excitation of $0.5-1 \mathrm{~Hz}$ surface waves in the sedimentary basin around Kamchatka river delta. Among processed records we have a few supporting each of these two ideas. The individual spectral anomalies of KRI and PET stations are consistent with earlier results from small earthquake amplitude data (FEDOTOv, 1972; ZoBIN et al., 1993) and $A_{\max }$ data (GUSEv et al., 1997a).

5. On the whole, regression curves describe data quite well and observed anomalies are genuine. At the present stage the data description can be considered as completed, but the results seem not to be sufficiently definitive to recommend them for engineering applications. 
The use of the average source spectrum is an alternative to the use of the average FSA when one must perform the stochastic modeling of engineering parameters of strong motions. In addition, a wide-band description of the source spectrum can shed light on the physics of an earthquake source. In this section we endeavor to construct a model of average source spectrum for Kamchatka. To accomplish this we use the inverse procedure: the source spectrum was calculated from the assumedly known Fourier spectrum at $R=100 \mathrm{~km}$.

By definition, the source spectrum is the spectrum of seismic moment rate $\dot{M}_{0}(t)$ for equivalent point source ("equivalent" means "producing similar far-field displacement amplitudes"). On this basis we used the following procedure for calculation of the high frequency part of the source spectrum. The estimated Fourier spectrum of acceleration for rock ground was reduced to $R=1000 \mathrm{~km}$ (far-field zone) by means of the formula similar to (4):

$$
\begin{aligned}
\log F S A\left(M_{W}, R=1000 \mid f_{K}\right)= & \log F S A_{\text {rock }}^{E}\left(M_{W}, R=100 \mid f_{K}\right) \\
& -\operatorname{Att}\left(M_{W}, R=100 \mid f_{K}\right) \\
& +\operatorname{Att}\left(M_{W_{i}}, R=1000 \mid f_{K}\right),
\end{aligned}
$$

where $F S A_{\text {rock }}^{E}\left(M_{W}, R=100\right)$-calculated above empirical Fourier spectrum for Kamchatka on rock; Att - the theoretical model of attenuation function. Subsequently the high frequency part of source spectrum may be calculated by means of the following formula:

$$
\dot{M}_{0}(f)=\frac{4 \pi \rho c_{S}^{3}}{(2 \pi f)^{2}} \cdot \frac{1000}{\exp \left(-\frac{\pi f \cdot 1000}{Q(f) c_{S}}\right)} \cdot F S A\left(M_{W}, R=1000 \mid f\right),
$$

where $1 /(2 \pi f)^{2}$ - the coefficient to convert acceleration spectrum to displacement spectrum, $\rho=2.7 \mathrm{~g} / \mathrm{cm}^{3}$ - density; $c_{S}=3.5 \mathrm{~km} / \mathrm{s}-S$-wave velocity; $Q(f)=$ $250 f^{0.8}-Q$-factor.

The low-frequency part of source spectrum, at $f<0.5 \mathrm{~Hz}$, was calculated by means of the Brune's model as modified by Boore $(1983)\left(c_{S}=3.5 \mathrm{~km} / \mathrm{s}\right.$ and $\Delta \sigma=40$ bar were accepted). On Figure 9 we plotted $f^{2} \dot{M}_{0}(f)$, or "acceleration source spectrum" (GUSEV, 1983). As seen on Figure 9, both parts match quite well without additional adjustment.

In their high-frequency part, empirical acceleration source spectra clearly show the " $f_{\max }$ " phenomenon (HANKs, 1982), or high-frequency cutoff. To determine the cutoff frequency value we have used the common " $-3 \mathrm{~dB}$ " definition: $f_{\max }$ equals the frequency where $f^{2} \dot{M}_{0}$ equals $0.7 \cdot\left(f^{2} \dot{M}_{0}\right)_{\max }$. The results for each of the magnitudes $M_{W}=5,6,7$ and 8, shown in Figure 9, clearly, illustrate the decrease of $f_{\max }$ with increased $M_{W}$, from $12 \mathrm{~Hz}$ at $M_{W}=5$ to $3 \mathrm{~Hz}$ at $M_{W}=8$. This observation suggests the source-related origin of $f_{\max }$ phenomenon that may be associated with a probable variation of some characteristic frequency of the source 
with magnitude (Gusev, 1983; PApageOrgiou and AKI, 1983). The near-station attenuation effects (ANDERSON and HoUGH, 1984) contribute to spectral cutoff formation as well, and the observed cutoff must be ascribed to the joint effect of both mentioned mechanisms.

Another possible explanation for $f_{\max }$ vs. $M_{W}$ dependence is that it is caused by nonlinear effects in near-station attenuation (the larger magnitude, the larger amplitude, the lower effective $Q$, the lower $f_{\max }$ ) (see e.g., AtKInSON and Silva, 1997; DURWARD et al., 1996). We consider this explanation as less probable for the following reason. As is well known, the typical acceleration level for nonlinear attenuation to manifest itself is of the order $A_{\max }=0.3-0.4 \mathrm{~g}$ for medium ground and 0.1-0.2 g for soft ground (BERESNEV and WEN, 1996); for rock ground, no observations of nonlinear spectral modification at high amplitude levels are known to us. Our data derive from rock and medium ground only, and maximum acceleration in our data set for medium ground is only $0.22 \mathrm{~g}$ (GUSEV et al., 1997a). Thus the expected nonlinear effects are minor, and hardly can explain the observed shift of $f_{\max }$ by four times.

Note also that possible errors of estimated source spectral shapes related to imperfect accounting for source finiteness effects are frequency-independent and thus cannot contribute to $f_{\max }$ vs. $M$ dependence. Another possible cause of error in spectral shapes is the error in accepted point source attenuation at short distances. This bias may be real (though limited); however it again cannot account for $f_{\max }\left(M_{W}\right)$ dependence.

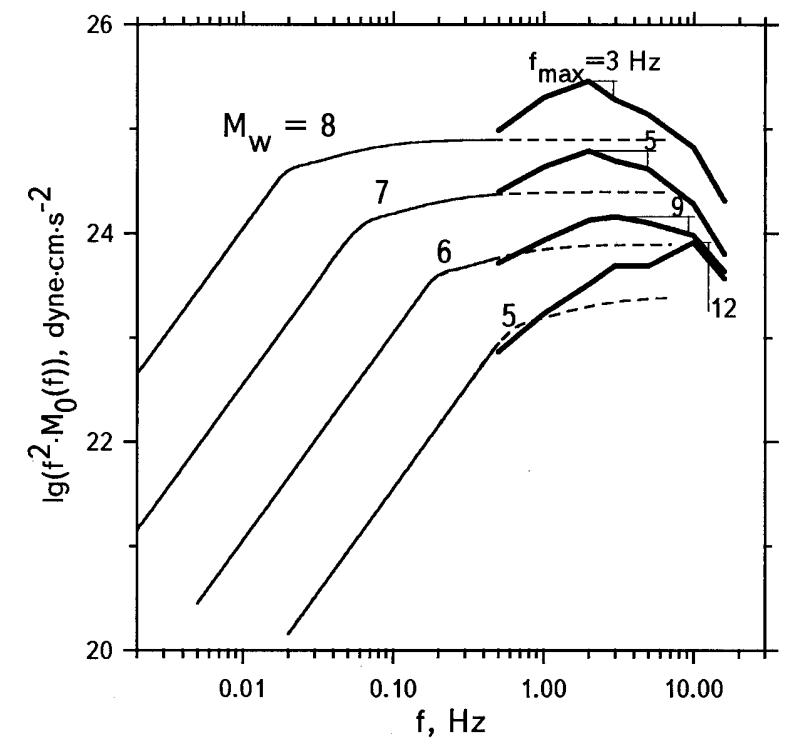

Figure 9

Estimated acceleration source spectrum scaling law for Kamchatka earthquakes. Thick line: short-period part derived from the empirical Fourier spectrum; thin line: Brune's spectrum with $\Delta \sigma=40$ bar; dashed line: Brune's spectrum continued to frequencies above $0.5 \mathrm{~Hz}$. 
1. The first version of the average relationship $\operatorname{FSA}\left(M_{W}, R\right)$ for horizontal ground motion of shallow earthquakes recorded on rock ground was determined for Kamchatka.

2. The general character of the determined relationship $F S A\left(M_{W}\right)$ does not contradict the known theoretical models and empirical relationships. At $M_{W}=7-$ 7.5, the absolute FSA level around $1 \mathrm{~Hz}$ is comparable for Kamchatka, Japan and California, and for $1-3 \mathrm{~Hz}$ is comparable for Kamchatka and Japan (both above the Californian level). Above 3-4 Hz, Kamchatka spectral level is somewhat below that for Japan. The distance attenuation model postulated in data analysis agrees well with actual data.

3. The spectral correction factors for KBG, PET and KRI stations have been determined. The previously found anomalies of $A_{\max }$ for PET and KRI stations agree well with the anomalies of Fourier spectra. With the site/station correction factors included, the residual error of the regression model is about $0.3 \log 10$ units, comparable to the values obtained in previous similar studies.

4. The results of the present analysis provide a compact description of the observed strong motion data set. To predict convincingly future ground-shaking for engineering purposes, additional analysis is needed. The data volume did not allow us to separate reliably the ground-type effect, individual site effect for an individual station, and the effect of a subregion.

5. The first version of the average earthquake source spectrum model for Kamchatka is proposed. These spectra manifest clear magnitude-dependent $f_{\max }$ features that may reflect the source-related contribution to the $f_{\max }$ phenomenon.

\section{Acknowledgements}

This work was supported by the Russian Foundation of Fundamental Research through Grant 97-05-65056 and Kamchatkan Regional Administration through Grant 19-95. The authors are indebted to Gail Atkinson for valuable comments which enhanced the paper.

\section{REFERENCES}

ABE, K. (1979), Size of Great Earthquakes of 1837-1974 Inferred from Tsunami Data, J. Geoph. Res. 84, $1561-1568$

Abybakirov, I. R., and Gusev, A. A. (1990), Estimation of Scattering Properties of Lithosphere of Kamchatka Based on Monte-Carlo Simulation of Record Envelope of a Near Earthquake, Phys. Earth Planet. Inter. 64, 52-67. 
Anderson, J. G., and Hough, S. (1984), A Model for the Shape of the Fourier Amplitude Spectrum of Acceleration at High Frequencies, Bull. Seismol. Soc. Am. 74, 1969-1994.

Atkinson, G. M. (1993), Source Spectra for Earthquakes in Eastern North America, Bull. Seismol. Soc. Am. 83, 1778-1798.

Atkinson, G. M., and Mereu, R. F. (1992), The Shape of Ground Motion Attenuation Curves in Southeastern Canada, Bull. Seismol. Soc. Am. 82, 2014-2031.

Atkinson, G. M., and Silva, W. (1997), An Empirical Study of Earthquake Source Spectra for California Earthquakes, Bull. Seismol. Soc. Am. 87, 97-113.

Beresnev, I. A., and Wen, K.-L. (1996), Nonlinear Soil Response-A Reality?, Bull. Seismol. Soc. Am. 86, 1964-1978.

Boore, D. M. (1983), Stochastic Simulation of High-frequency Ground Motions Based on Seismological Models of the Radiated Spectra, Bull. Seismol. Soc. Am. 73, 1865-1894.

Brune, J. N. (1970), Tectonic Stress and the Spectra of Seismic Shear Waves from Earthquakes, J. Geophys. Res. 75, 4997-5009.

Durward, J. A., Boore, D. M., and Joyner, W. B. (1996), The Amplitude Dependence of High-frequency Spectral Decay: Constraint on Soil Nonlinearity, Proc. of the International Workshop on Site Response Subjected to Strong Earthquake Motions, Yokosuka Japan, vol. 2, 82-103.

Fedotov, S. A., Energy Classification of the Kamchatka-Kuriles Earthquakes and Problem of Magnitudes (Nauka, Moscow 1972) (in Russian).

Fukushima, Y., and Tanaka, T. (1990), A New Attenuation Relation for Peak Horizontal Acceleration of Strong Earthquake Ground Motion in Japan, Bull. Seismol. Soc. Am. 80, 757-783.

Gusev, A. A. (1983), Descriptive Statistical Model of Earthquake Source Radiation and its Application to Short-period Strong Motion, Geophys. J. Roy. Astr. Soc. 74, 787-808.

Gusev, A. A. (1989), Multiasperity Fault Model and the Nature of Short-period Subsources, Pure appl. geophys. 130, 635-660.

Gusev, A. A. (1990), A preliminary version of design seismic load for Petropavlousk-Kamchatsky. In Voprosy Inzhenernoi Seismologii, iss. 31 (Nauka, Moscow 1990) pp. 67-85 (in Russian).

Gusev, A. A. (1991), Intermagnitude Relationship and Asperity Statistics, Pure appl. geophys. 136, $515-527$.

Gusev, A. A., and Melnikova, V. N. (1990), Relations between Magnitudes: Global and Kamchatka Data, Volc. Seis. \# 6, 55-63 (in Russian).

Gusev, A. A., and Petukhin, A. G. (1996), An Algorithm for Forecasting Earthquake Ground Motion, Volc. Seis. 17, English edition 571-584.

Gusev, A. A., Gordeev, E. I., Guseva, E. M., Petukhin, A. G., and Chebrov, V. N. (1997a), The First Version of the $A_{\max }\left(M_{W}, R\right)$ Relationship for Kamchatka, Pure appl. geophys. 149, 299-312.

Gusev, A. A., Petukhin, A. G., Guseva, E. M., Gordeev, E. I., and Chebrov, V. N. (1997b), Scaling Fourier Spectra of Strong Ground Motion Recorded on Kamchatka in Terms of Distance, Moment Magnitude and Other Factors, 29th General Assembly of IASPEI, Thessaloniki, Greece, August 18-28. Abstracts, 35 pp.

Gusev, A. A., Guseva, E. M., Petukhin, A. G., Gordeev, E. I., and Chebrov, V. N. (1998), Peak Ground Accelerations from Data of Strong Motion Instruments in Kamchatka, Physics of the Earth 4, $20-27$.

Guseva, E. M., Gusev, A. A., and Oskorbin, L. S. (1989), A Program Package for Digital Processing of Seismic Records and its Testing Using Some Strong Motion Records, Volc. Seis. \# 1, 35-49.

HanKs, T. C. (1982), $f_{\max }$, Bull. Seismol. Soc. Am. 72, 1867-1879.

Kamiyama, M., and Matsukawa, T. (1990), An Empirical Scaling of Strong-motion Spectra with Application to Estimate of Source Spectra, Structural Engineering and Earthquake Engineering, Proc. of Japan Soc. Civil Engineering 76, 331-342.

Papageorgiou, A. S., and AKI, K. (1983), A Specific Barrier Model for the Quantitative Description of Inhomogeneous Faulting and the Prediction of Strong Ground Motion-1. Description of the Model, Bull. Seismol. Soc. Am. 73, 693-722.

Trifunac, M. D. (1976), Preliminary Empirical Model for Scaling Fourier Amplitude Spectra of Strong Acceleration in Terms of Earthquake Magnitude, Source-to-station Distance, and Recording Site Conditions, Bull. Seismol. Soc. Am. 66, 1343-1373. 
Trifunac, M. D. (1989), Dependence of Fourier Spectrum Amplitudes of Recorded Earthquake Accelerations on Magnitude, Local Soil Conditions and on Depth of Sediments, Earthquake Eng. Struct. Dyn. 18, 999-1016.

Trifunac, M. D., and LeE, V. W. (1990), Frequency Dependent Attenuation of Strong Earthquake Ground Motion, Soil Dyn. and Earthquake Eng. 9, 3-15.

VANMARKe, E. H. (1986), Random vibration approach to seismic safety evaluation. In Random Vibration (Elsevier Science Publishers B.V. 1986) pp. 499-509.

Zobin, V. M., Fedotov, S. A., Gordeev, E. I., Guseva, E. M., and Mityakin, V. P. (1988), Large Earthquakes in Kamchatka and Commander Islands in 1961-1986, Volc. Seis. \# 1, 3-23 (in Russian).

Zobin, V. M., Mityushrina, S. V., and Chirkova, V. N. (1993), Station Corrections to Fedotov's Nomogram for the Energy Classification of Kamchatka and Commander Islands Earthquakes, Volc. Seis. \# 1, 82-92 (in Russian).

(Received April 23, 1998, accepted January 23, 1999) 